\title{
Micropatterning Co-cultures of Epithelial Cells on Filter Insert Substrates
}

\author{
Ana C. Paz ${ }^{1,2, \#, ~ S a h a r ~ J a v a h e r i a n ~}{ }^{1, \#}$ and Alison P. McGuigan ${ }^{1,2, *}$ \\ ${ }^{I}$ Department of Chemical Engineering and Applied Chemistry, University of Toronto, 200 College St. Toronto, ON, M5S \\ 3E5, Canada \\ ${ }^{2}$ Institute of Biomaterials and Biomedical Engineering, University of Toronto, Ontario, Canada
}

\begin{abstract}
A key feature of mature epithelium is the presence of an apical-basal polarization. In vitro this is achieved by culturing either pure populations of epithelial cells or unorganized mixtures of primary cell populations harvested from epithelial tissues in a filter insert culture system. In addition to epithelial cells however, multiple other cell types are also present in normal epithelium in a specific organization. Generally the organization of component cells within a tissue is critical for normal function. The importance of the organization of different cell populations within normal and diseased epithelium in vitro has received little attention, in part due to the lack of available methods for spatially controlling multiple cell populations while maintaining the culture conditions necessary to generate polarized and mature epithelium. We have developed a set of micropatterning techniques to spatially control the organization of co-cultures of epithelial cells on filter insert membranes under the culture conditions necessary to induce epithelial cell polarization. We describe a fibronectin microprinting method that allows arbitrary pattern generation and a parafilm insert method that allows only simple stripe geometry patterning but does not require the photolithographic equipment that the fibronectin microprinting method requires, and can be adopted by any epithelial biology lab. We also demonstrate that our micropatterning methods do not alter the formation of cilia, a marker of epithelium maturation. Our methods provide a novel tool for studying epithelial biology in polarized epithelial sheets containing multiple cell populations with controlled organization.
\end{abstract}

Keywords: Epithelial cells, micropatterning, co-culture, polarization, filter insert culture, microcontact printing.

\section{INTRODUCTION}

Epithelial cells line most of the major organs of the body as sheets of epithelial tissue. Engineering functional epithelial sheets is therefore important for generating functional artificial organ replacements that require an epithelial component [1,2]. Furthermore, about $90 \%$ of human cancers arise in epithelial tissues [3]; therefore realistic in vitro models of epithelium could provide a valuable tool for better understanding mechanisms of early cancer development and other epithelial diseases. Generating appropriately organized epithelial tissue in vitro, similar to that of native epithelium, is important for engineering artificial epithelium and for establishing relevant in vitro models because cell organization within a tissue typically is a crucial factor for determining tissue function and behavior [4]. Native epithelial sheets consist of a layer of polarized epithelial cells [5]. Apical-basal polarization of the epithelial cells in the sheet is a key requirement for developing functional and mature epithelium [6]. In vitro, polarized epithelium is often generated in $2 \mathrm{D}$ culture using the filter insert culture system [7], and in 3D culture by embedding epithelial cell aggregates in biopolymer gels to generate cysts comprised of polarized epithelial cells [8]. Both these

*Address correspondence to this author at the Department of Chemical Engineering and Applied Chemistry, University of Toronto, 200 College St. Toronto, ON, M5S 3E5, Canada; Tel: 416978 7552;

E-mail: alison.mcguigan@utoronto.ca

"These authors contributed equally to this work in vitro culture methods use either pure populations of epithelial cells or unorganized mixtures of primary cell populations harvested from epithelial tissues. In addition to epithelial cells however, multiple other cell types such as goblet and basal cells are also present in normal epithelium and are critical for normal epithelium function such as mucous production. In the case of diseased epithelium multiple cell populations are also present since the epithelial sheet contains a localized region of diseased cells. The importance of the organization of different cell types within normal and diseased epithelium in vitro has received little attention, in part due to the lack of available methods for spatially controlling multiple cell populations while maintaining the culture conditions necessary to generate polarized and mature epithelium. Co-patterning techniques exist for generating controlled localization of distinct cell types on glass and plastic culture surfaces [9], however these techniques have not been translated for use with epithelial cells cultured using the filter insert culture system. We therefore set out to adapt solid substrate patterning methods to enable co-patterning of multiple epithelial cell populations on filter insert membranes. Our methods allow us to spatially control epithelial cell organization in co-culture while maintaining the culture conditions required for epithelial cell apical-basal polarization and maturation.

A key feature of mature, functional epithelium is the presence of apical-basal polarization [10]. An epithelial cell becomes apically-basally polarized due to structural changes that take place in the cell in response to different 
environmental and internal clues. Both cell-cell contact (required to form tight junctions) and cell-basement membrane interactions play an important role in the establishment of the apical-basal polarity [8]. Currently, one of the most common in vitro model systems used to study polarized epithelium tissue is the filter insert culture system. During filter insert culture the cells are grown as a $2 \mathrm{D}$ (or multilayer 3D) sheet on the top surface of a filter insert and are initially supplied with nutrients from culture medium situated in both the top compartment of the filter insert and the bottom compartment situated below the porous membrane of the filter insert. Cells are usually grown for few days under these conditions to ensure they are confluent before inducing complete apical-basal polarization and epithelium maturation. Cell maturation is induced by removal of the serum-containing medium in the top compartment and replacement either with nothing (i.e. Air interface culture) or with serum-free medium. Cells continue to be supplied with nutrients at their basal surface from culture medium in the chamber beneath the filter insert [11]. Apical-basal polarization in cells cultured in the filter insert system is thought to be encouraged by the ability of cells to access nutrients from their basal side [12]. Cells apicallybasally polarize typically over a 10-20 day period [13], depending on cell type [14]. Typical markers of apical-basal polarization include ZO-1 expression at the apical surface of the cell-cell membranes [7] and the development of cilia from the apical surface of the cells $[15,16]$.

Systems allowing co-culture of epithelial cells with a different cell population are useful for studying the effect of other cells types on epithelium maturation, polarization, and function, as well as the affect of epithelium on the functional phenotype of the second cell population. For example, coculture experiments using fibroblast and stromal cells with epithelial cells have demonstrated that the fibroblasts or stromal cells enhanced morphological polarization as well as advanced differentiation of the epithelial cells [17, 18]. It has been also established in an in vitro co-culture system that wound healing in epithelium co-cultured with intestinal myofibroblast progressed more efficiently than in pure cultures where no myofibroblasts were present [19]. Unfortunately, in vitro co-culture studies involving polarized epithelium have been largely limited to co-culture of two cell populations in different chambers of the filter insert culture system $[20,21]$. These studies limit the influence of one cell type on another to the role of diffusible factors, and completely leave out the role of direct cell-cell interactions. Only limited reports have focused on the effect of contactdependent co-cultures of epithelial cells [22, 23]. These studies, however, did not provide adequate conditions for complete polarization of epithelium and did not control cell organization. To better address such biological questions, we therefore set out to develop a set of methods that allows controlled co-culture of epithelial cells with other cell types under conditions suitable for complete apical-basal polarization and maturation of epithelium.

Currently the simplest and most common methods for micropattening cells are microfluidic patterning [24], microcontact printing, and the use of microstencils [25, 26]. All of these methods have been designed for micropatterning cells on glass or plastic substrates. Of these methods the last two are most adaptable for use on filter insert membranes and provided a starting point for developing our patterning strategies. Microcontact printing utilizes an elastomeric stamp or mould, usually made of poly(dimethylsiloxane) (PDMS), prepared by casting the liquid prepolymer of an elastomer against a master, fabricated using photolithography, which has a patterned relief structure [27]. This patterned stamp is then coated with a desired adhesion protein and brought into contact with a cell-repellent substrate. When the stamp is removed, a pattern of the adhesive protein remains on the substrate. Cells preferentially adhere to substrate regions containing the adhesive protein. To generate patterned co-cultures, the first cell type is seeded in serum free medium and allowed to adhere to the microstamped proteins. Then a second cell type is seeded in serum-containing medium and adheres to the locations where the first cell type is not present [25].

Stencil techniques involve generating thin PDMS [28] membranes containing holes of a desired pattern. The PDMS membrane is reversibly bonded to a desired cell adhesive substrate. Cells cannot adhere in regions where PDMS is present but can adhere in the "hole regions" where the underlying cell adhesive substrate is exposed. At any desired time the PDMS layer can be removed to leave a patterned substrate with cells located only where a PDMS hole was previously present. A second cell type can then be seeded to generate patterned co-cultures [29].

Both of these existing methods require access to a clean room and specialized equipment to generate a photolithographic master. Neither of these patterning strategies has been used previously to generate patterned co-cultured epithelial cell sheets for subsequent filter insert culture and apicalbasal polarization. Furthermore, it is not clear how well the patterned co-cultures produced using these strategies can be maintained over time, which is an important consideration for epithelial cell applications where an extended culture period is required to induce apical-basal cell polarization. We set out to adapt existing soft-lithography strategies for micropatterning co-cultures of epithelial cells on filters insert membranes. Specifically, we have adapted the extracellular matrix (ECM) protein stamping technique to generate patterned co-cultured epithelial sheets and developed a stencil type method using Parafilm inserts that does not require a clean room or complex equipment. Our patterning methods provide a set of tools for patterning epithelial cell populations while maintaining the culture conditions necessary to generate polarized epithelium.

\section{METHODS}

\section{Cell Culture}

We used the ARPE-19 human retinal epithelial cell line (ATTC CRL-2302, USA) and the MDCK dog kidney epithelial cell line (ATTC CCL-34, USA) at passages between P10-20. For generating co-patterns we used wild type ARPE-19 cells or MDCK cells and ARPE-19 cells or MDCK cells infected with a lentivirus encoding eGFP. ARPE-19 cells were grown in Dulbecco's modified Eagle's medium/nutrient F-12 (DMEM/F-12, Invitrogen, Canada) supplemented with $10 \%$ fetal bovine serum (FBS) (Sigma Aldrich, Canada) and 1\% Penicillin/Streptomycin (VWR, Canada) and maintained in 
an incubator at $5 \% \mathrm{CO}_{2}$. MDCK cells were grown in Dulbecco's modified Eagle's medium (DMEM, Biowhittaker, Canada) supplemented with $10 \%$ FBS and $1 \%$ Penicillin/ Streptomycin and maintained in an incubator at $5 \% \mathrm{CO}_{2}$

\section{MASTER AND PDMS STAMP FABRICATION}

We created an SU-8 master containing specific topographic features as described previously [30]. Briefly, we designed a photomask with the desired geometric patterns (200 $\mu \mathrm{m}$-wide stripes) using AutoCad and printed the pattern in high-resolution black ink on a transparent sheet. We exposed negative photoresist (MicroChem Corp, USA), spin-coated onto a clean $3 \times 5$ glass slide, to UV light through our mask to transfer the geometric patterns from the photomask to the photoresist. We then washed away unexposed photoresist with a developer solution (MicroChem Corp, USA) to generate a master with the desired topographic features. The master was silanized under vacuum for 3 hours using 1-Trichlorosilane (UCT, USA)). We generated a PDMS stamp by replica molding as described previously [30]. Briefly, we poured a degassed 10:1 (w/w) mixture of PDMS polymer:curing agent ((Dow Corning Corp, USA) over our master and cured at $60^{\circ} \mathrm{C}$ for 3 hours. The hardened PDMS stamp was then peeled off from the master.

\section{PARAFILM INSERT FABRICATION}

To generate parafilm inserts to fit into filters inserts for a 6-well plate we cut circular pieces of Parafilm $(150 \mathrm{~mm}$ in radius).To generate a stripe pattern in the parafilm inserts we cut arrays of parafilm using surgical blades (No 10, Feather, Japan). The cut parafilm pieces were removed using tweezers leaving behind holes of rectangular shape in the parafilm inserts. To bind the parafilm inserts onto the filter insert membrane we pressed the parafilm insert firmly onto the filter insert surface to ensure full contact and a good seal with the substrate material. We then placed the insert on a hot plate set at $50{ }^{\circ} \mathrm{C}$ for 5 seconds to further strengthen the bond between parafilm and the membrane. We then added 1 $\mathrm{mL}$ of phosphate buffer saline (PBS, VWR, Canada) into each well and degassed the films for 5 minutes at a pressure of $30 \mathrm{psig}$. This step was critical to ensure liquid infiltration into the small holes and subsequent cell patterning. We UV sterilized the films in PBS for 20 minutes and then removed the PBS from the wells before cell culture. We then added FBS to the filters and incubated at $37^{\circ} \mathrm{C}$ for 30 minutes prior to seeding of the first cell type.

\section{TRANSWELL PATTERNING STRATEGY}

Fig. (1) outlines our two strategies for patterning two populations of epithelial cells on filters insert membranes. Using both methods we generated co-patterns of wild-type and GFP expressing cells (using MDCK and GFP MDCK cells or ARPE-19 and GFP-ARPE-19 cells). All methods used filter insert membranes with $0.4 \mu \mathrm{m}$ pores (Corning, USA). Our first strategy (Fig. 1a) is an adaptation of cell patterning techniques carried out by controlled deposition of ECM proteins [27, 31]. This strategy makes use of a PDMS stamp generated by soft-lithography [32] by replica molding the stamp over topographic features generated using photolithography. To generate patterned co-cultures on the filters insert membrane using this fibronectin microprinting method, first a $0.5 \mathrm{~cm} \times 0.5 \mathrm{~cm}$ PDMS stamp with $200 \mu \mathrm{m}$ wide stripes pattern arrays was sterilized by submerging the stamp in $70 \%$ ethanol solution for at least $30 \mathrm{~min}$ and dried with air. Then, the stamp was placed in a sterile Petri dish with the pattern side up, covered with $20 \mu$ l of $100 \mu \mathrm{g} / \mathrm{mlfibronectin}$ solution (Sigma Aldrich, Canada), and incubated for one hour at room temperature. Excess fibronectin not adsorbed onto the stamp was then carefully removed by blotting the stamp on a kimwipe and rinsing it once with double distilled water. The stamp was then blotted again on a kimwipe until there was no visible liquid left on the stamp. Immediately after drying, the inked stamp was placed for $10 \mathrm{~min}$ on the filter insert membrane, and a $6 \mathrm{~g}$ weight was placed over the stamp to ensure conformal contact. After 10 minutes the weight was removed, a PDMS frame was placed around stamp (this frame was later used to contain the cell seeding solution), and the stamp was removed with tweezers. A $0.05 \%(\mathrm{~W} / \mathrm{V})$ BSA (Sigma Aldrich, Canada) solution was then added over the deposited fibronectin pattern and incubated for one hour at $37^{\circ} \mathrm{C}$. Finally, the BSA solution was removed and the membrane was rinsed with serum-free medium.

To seed the first GFP expressing cell type (GFP MDCK or GFP ARPE-19) we prepared a cell suspension of $2 \times 10^{6}$ cells $/ \mathrm{mL}$ in serum-free medium and placed $500 \mu \mathrm{L}$ of the suspension over the fibronectin pattern. The sample was incubated at $37{ }^{\circ} \mathrm{C}, 5 \% \mathrm{CO}_{2}$ for 1.5 hours for ARPE- 19 and 2.5 hours for MDCK cells. Next, un-adhered cells were removed by rinsing the filter insert membrane with serumfree medium. We added $1 \mathrm{ml}$ of serum-free medium to the bottom compartment of the filter insert culture system to better visualize the generated patterns on the filter insert membrane by microscopy. To generate patterned co-cultures the sample was incubated with FBS for $20 \mathrm{~min}$ at $37{ }^{\circ} \mathrm{C}$ to deactivate the BSA blocking before adding the second cell type. We then prepared a cell suspension (of the second cell type, wild type MDCK or ARPE-19) of $3 \times 10^{6}$ cells $/ \mathrm{mL}$ in serum medium and added $500 \mu \mathrm{L}$ of it to the sample and incubated at $37{ }^{\circ} \mathrm{C}, 5 \% \mathrm{CO}_{2}$ for 2 hours for ARPE-19 and 3 hours for MDCK cells. Un-adhered cells were then removed by rinsing the filter with serum medium. Finally, we added $1.5 \mathrm{ml}$ of serum medium to the top compartment and $2.6 \mathrm{ml}$ of serum medium to the bottom compartment. On the next day we switched the medium in the top compartment to serum-free medium and continued the culture for up to 2 weeks periodically supplying the cells with fresh medium in both compartments.

Fig. (1b) shows our alternative more simple strategy for co-patterning that uses parafilm inserts. While this parafilm strategy is limited to only stripe geometric patterns and is not suitable for applications where features smaller than $100 \mu \mathrm{m}$ are desired [33], this method is significantly easier to optimize than the microprinting technique, does not require use of a photolithographic master, and the geometry is adequate to answer a wide range of questions requiring spatially organized epithelium. To generate patterned cocultures using the Parafilm insert method we placed a parafilm insert containing $300 \mu \mathrm{m}$ stripe arrays into the upper chamber of the filter insert and firmly bonded the parafilm insert to the filter insert membrane (see below). To seed the first cell type (GFP MDCK or GFP ARPE-19) we added $500 \mu \mathrm{L}$ of a cell suspension of $2 \times 10^{6}$ 

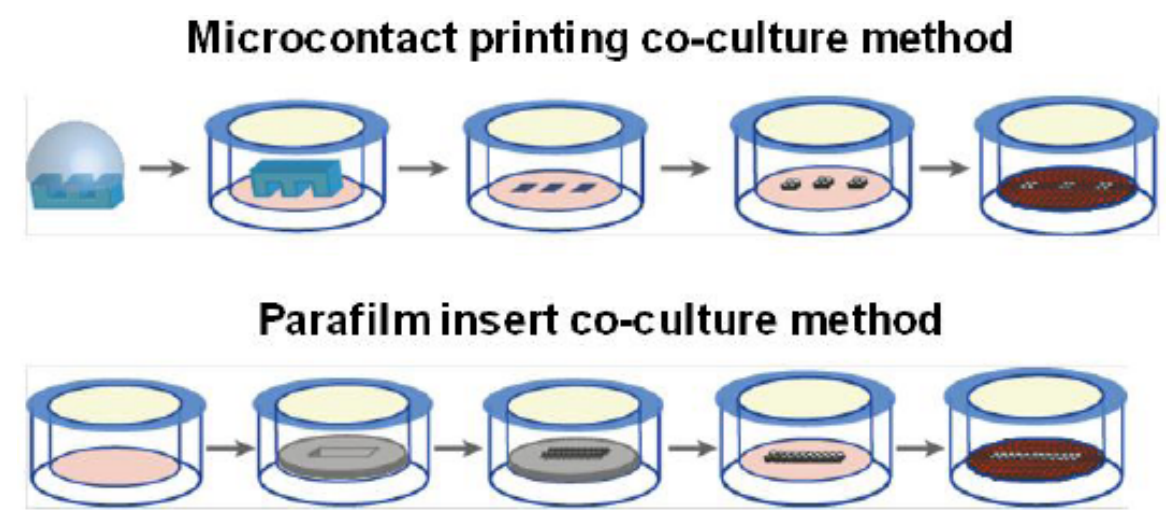

Fig. (1). Schematic of patterning strategies.

cells/mL making sure that the entire surface of the insert was covered by the cell suspension. We incubated the samples at $37{ }^{0} \mathrm{C}, 5 \% \mathrm{CO}_{2}$ for 3 hours. Next, we removed the cell suspension and gently washed away any un-adhered cells with our cell culture medium. To generate the co-cultures, we removed the parafilm inserts 3 hours after seeding of the first cell type for MDCK and 12 hours for ARPE-19 and incubated the patterned cells in fetal bovine serum for 20 minutes, at $37{ }^{\circ} \mathrm{C}$. We next removed the FBS and seeded $500 \mu \mathrm{L}$ of a second cell type (MDCK or ARPE-19) at a seeding density of $3 \times 10^{6}$ cells $/ \mathrm{mL}$. We incubated the patterns for 1 hour to allow the additional cells to adhere to the surface free from the first cell type and then thoroughly washed the substrates with growth medium to remove any un-adhered cells. Finally, we added $1.5 \mathrm{~mL}$ of serum medium to the top compartment and $2.6 \mathrm{~mL}$ of serum medium to the bottom compartment. On the next day we switched the medium in the top compartment to serum-free medium and continued the culture for up to 2 weeks periodically supplying the cells with fresh medium in both compartments.

\section{FILTER INSERT CULTURE CONDITIONS}

We cultured the patterned co-cultures overnight, for one week or for two weeks. In the case of the one and two week cultures, to generate a full-polarized epithelial sheet we cultured the cells with serum medium in the top and bottom compartments of the filter insert culture system for 1 day and then replaced the medium in the top compartment with serum-free medium for the further days of culture. To generate un-patterned control samples for comparison we seeded $2 \times 10^{6}$ cells $/ \mathrm{mL}$ onto filter insert and incubated overnight with serum medium in the top and bottom compartments of the filter insert culture system. After one day we replaced the medium in the top compartment with serum-free medium and cultured the cells for one or two weeks.

\section{ASSESSING PATTERNED EPITHELIAL SHEETS}

To characterize the distribution of the two cell types on the filter inserts over time we stained co-cultures with DAPI (Invitrogen, Canada) and observed the GFP signal from the cells expressing GFP.To determine if the co-patterned cells undergo the same expected apical-basal polarization as is seen in un-patterned cultures we assessed one of the typical apical-basal polarization markers, formation of primary cilia, by staining the samples for acetylated $\alpha$ tubulin. We prepared samples for analysis by fixing cells in $4 \%$ paraformaldehyde (Electron Microscopy Sciences, USA), permeabilizing using $0.1 \%$ tween and staining with DAPI. We used monoclonal anti-acetylated $\alpha$ tubulin raised in mouse (1:1000, Sigma Aldrich, Canada) and Alexa 647-conjugated anti-mouse IgG (1: 250, Santa Cruz Biotechnology, USA) or Cy3-conjugated anti-mouse IgG (1: 250, Millipore, USA). Images were taken on an Olympus IX81 microscope.

\section{RESULTS}

\section{Patterned Co-cultures}

Here we present two methods for co-patterning epithelial cell populations on filter insert membranes. Firstly we generated a method to co-culture two population of cells by adapting a combination of two previously developed methods for microprinting ECM protein on rigid substrates for generating cell patterns [31] and a co-culture method using microstencils [25]. This method allows generation of any arbitrary size and shape pattern. We also devised a method to co-culture cells into simple stripe patterns using parafilm inserts, which although simplistic is adequate for a number of applications and can be performed without access to specialized equipment. Fig. (2) shows co-patterned MDCK/GFP MDCK and ARPE-19/GFP ARPE-19 epithelial cell sheets, zero days after patterning, generated using our adapted fibronectin microprinting and our Parafilm insert methods. As expected, both methods resulted in patterns of alternating stripes of GFP and non-GFP cells. For the fibronectin microprinting method the stripes were about 200 $\mu \mathrm{m}$ in width for the GFP cells and $500 \mu \mathrm{m}$ for non-GFP cells. For the parafilm insert method the GFP cells stripes were about $250 \mu \mathrm{m}$ and non-GFP cell stripes about $400 \mu \mathrm{m}$. As the parafilm inserts are generated manually the accuracy and precision of the stripe sizes are not as high as that of fibronectin microprinting method.

Selecting the correct cell density for seeding and the correct timing for the incubation period was critical for achieving robust patterning in each of the methods and with each cell type. For example, in the fibronectin microprinting method for MDCK cells, an initial seeding density of $2 \times 10^{6}$ cells $/ \mathrm{mL}$ with an incubation period of 2.5 hours before addition of the second cell type produced good patterning of the cells, and for ARPE-19 cells an initial seeding density of 

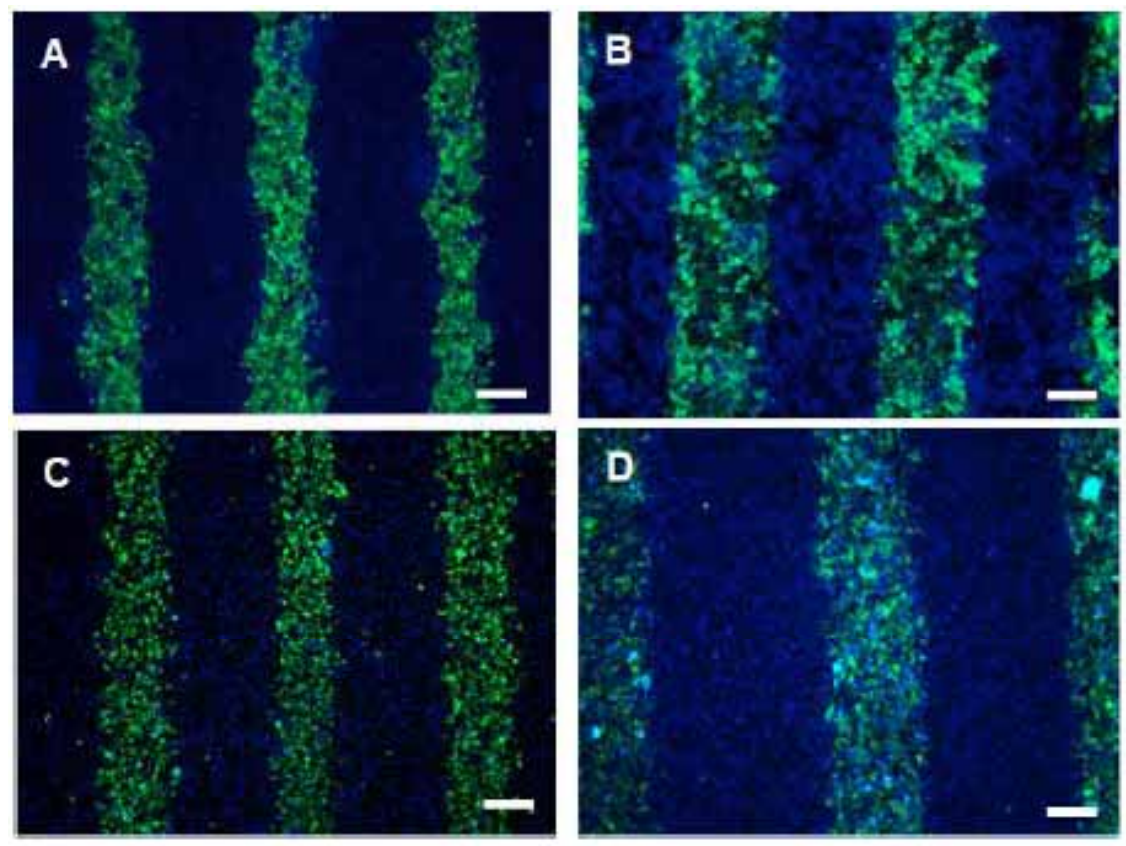

Fig. (2). Co-patterning of epithelial cells on filter insert membranes at day 0 after patterning. GFP expressing and non-GFP cells were patterned using our two described methods. A - Co-culture of GFP MDCK and wild type MDCK cells generated by fibronectin microprinting method. B - Co-culture of GFP MDCK and wild type MDCK cells generated by parafilm insert method. C - Co-culture of GFP ARPE-19and wild type ARPE-19 cells generated by fibronectin microprinting method. D - Co-culture of GFP ARPE-19 and wild type ARPE-19 cells generated by parafilm insert method. The scale bar is $200 \mu \mathrm{m}$ wide. Blue - dapi, Green - GFP.

$2 \times 10^{6}$ cells $/ \mathrm{mL}$ with an incubation period of 1.5 hours produced good patterning. Also, the incubation of the filter with FBS for 20-30 min before the addition of the second cell type was crucial to promote the attachment of the additional cells on the filter insert membranes. We found that although the fibronectin microprinting method allowed arbitrary pattern generation, stamping on the filter insert membrane was more difficult than on glass or tissue culture substrates. It was, therefore, very important to remove the excess fibronectin by blotting the stamp on kimwipe paper, instead of drying the stamp with air or nitrogen gas [27]. Also, the stamping step had to be done quickly so the inked stamp did not completely dry. After the stamp was placed on the filter it was important not to attempt to move the stamp until after the stamping period was complete. All the procedures were done with the filter insert placed in the well plate holder.

For the parafilm insert method the critical step in ensuring the successful generation of patterns was bonding of the parafilm insert to the filter membrane. Here, it is crucial to ensure the strongest possible bonding by applying pressure without disturbing the patterns in the parafilm insert. We found that the degassing step provided a good indication of whether the bonding between parafilm and the membrane was sufficiently strong. In the case of inadequate bonding the parafilm insert detached from the membrane under vacuum during the degassing step.

\section{PATTERN MAINTENANCE}

We wanted to assess the stability of the co-patterns over time since epithelial cells require 10-20 days to mature fully and it would be desirable to preserve the generated patterns for the relevant duration. Figs. (3 and 4) show maintenance of patterns over long time periods of culture (7-15 days) with serum free medium in the top chamber. Using both techniques MDCK cell sheets remained clearly patterned after 14 days in culture suggesting limited cell mobility within the polarized epithelial sheet. However, with the fibronectin microprinting method the patterns appear to change over time (Fig. 3) more than in samples patterned using the parafilm insert method (Fig. 4). ARPE-19 cells moved more significantly over the 15-day period and significant pattern distortion was observed when using both patterning techniques (Fig. 3C,D, Fig. 4B). This is most pronounced in the fibronectin microprinting method, where even by day 7 the GFP expressing cells mix with the wild type cells. ARPE-19 cell pattern fidelity was lower than in MDCK cells with the parafilm insert method as well, where by day 15 the pattern, while still distinguishable, was not sharp and the two cell populations had mixed.

\section{ASSESSING APICAL-BASAL POLARIZATION ON THE FILTER INSERT}

To ensure micropatterning the cells did not alter their subsequent apical-basal polarization on the filters we stained acetylated $\alpha$-tubulin (marker of cilia), which is a typical marker of apical-basal polarization [7, 34]. Since apicalbasal polarization is very well characterized in MDCK cells $[14,16,35,36]$, we used them for these experiments. Fig. (5) compares the development of cilia in patterned or unpatterned MDCK cells cultured for 7 or 15 days. In all samples cilia were visible by day 7 and appeared to have increased in length in our images taken at day 15. The frequency of cilia expression did not appear to vary between 

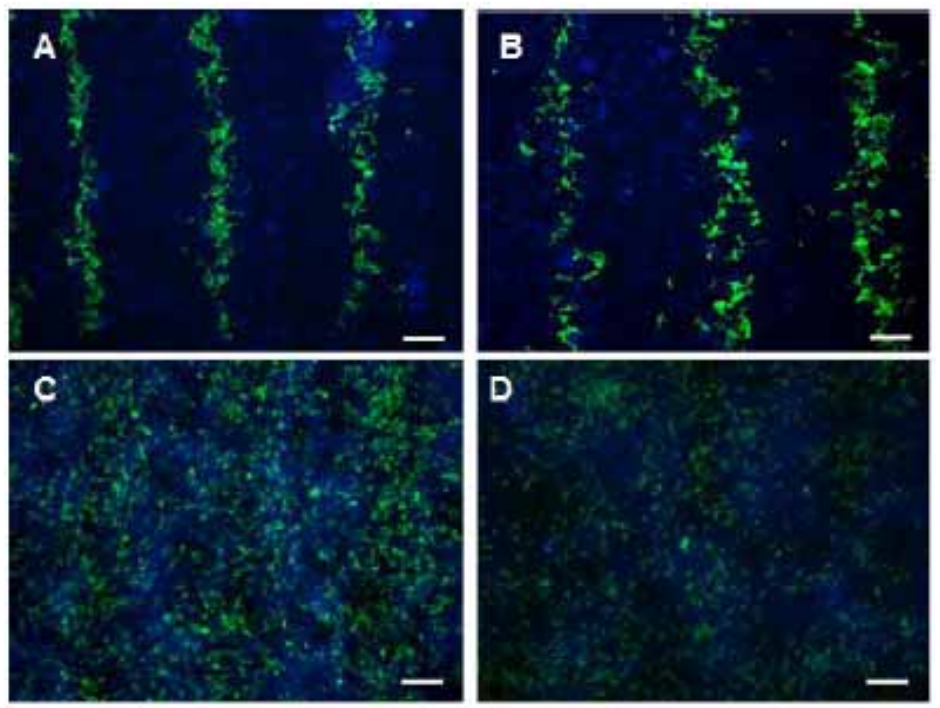

Fig. (3). Assessment of Fibronectin microprinted co-patterned epithelial sheets on filter insert membranes over time. All patterns were generated using the fibronectin microprinting method A - Co-culture of MDCK cells, day 7. B - Co-culture of MDCK cells, day 15. C - Coculture of ARPE-19 cells, day 7. D - Co-culture of ARPE-19 cells, day 15. The scale bar is $200 \mu \mathrm{m}$ wide. Blue - dapi, Green - GFP.
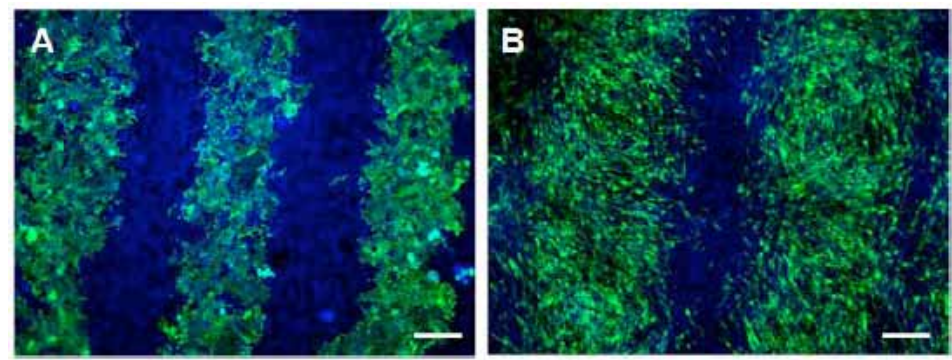

Fig. (4). Assessment of parafilm insert co-patterned epithelial sheets on filter insert membrane filters over time. All patterns were generated using the parafilm insert method A - Co-culture of MDCK cells, day 15. B - Co-culture of ARPE-19 cells, day 15 . The scale bar is $200 \mu \mathrm{m}$ wide. Blue - dapi, Green - GFP.
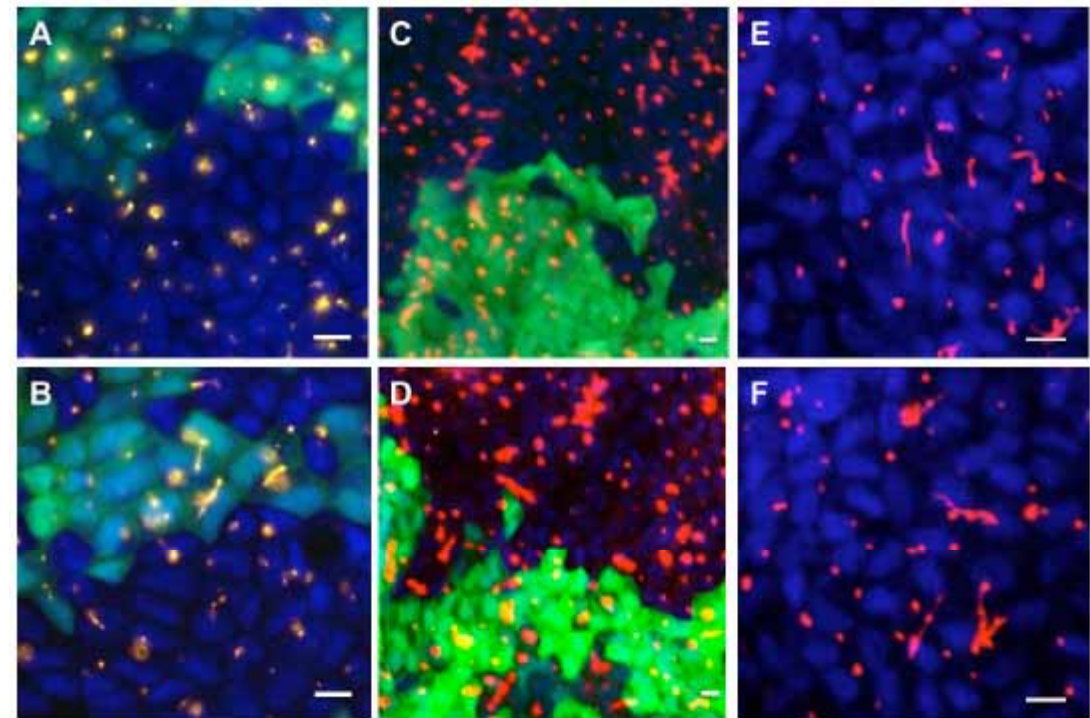

Fig. (5). Cilia expression (marked by acetylated $\alpha$-tubulin) in un-patterned versus co-patterned MDCK cells. A - Co-culture of MDCK cells generated by fibronectin microprinting method, day 7. B - Co-culture of MDCK cells generated by fibronectin microprinting method, day 15 . C - Co-culture of MDCK cells generated by parafilm insert method, day 7. D - Co-culture of MDCK cells generated by parafilm insert method, day 15. E -Un-patterned MDCK cell sheet, day 7. F- Un-patterned MDCK cell sheet, day 15. Blue - dapi, Green - GFP, Red Acetylated $\alpha$-tubulin 
the patterned and un-patterned samples and we quantified an average of approximately 25 cilia present per a $50 \mu \mathrm{m} \times 50$ $\mu \mathrm{m}$ square of surface.

\section{DISCUSSION}

Cell patterning is a strategy to spatially control the location of multiple cell populations on a cell culture substrate. By patterning cells into distinct locations within a sheet it is possible to probe the influence of mixing multiple cell populations on the structure and function of the generated epithelial tissue. The influence of different cell types within normal and diseased epithelium on tissue structure and function in vitro is not well characterized, in part due to the lack of available methods for spatially controlling multiple cell populations while maintaining the culture conditions necessary to generate polarized and mature epithelium. Here we report on the development of a set of tools to enable co-culture micropatterning of epithelial cells into patterns with controlled dimensions, while maintaining the filter insert culture conditions required for epithelial cell complete apical-basal polarization and maturation. Our in vitro techniques are potentially valuable tools for developing assays to study epithelial tissue properties and pathology. For example, using our system it would be possible to investigate how breast cancer cells behave beside normal breast epithelial cells and characterize the effect the tumor cells have on the surrounding normal epithelium tissue. Using our co-culture system it is also possible to characterize the influence of different cell types on epithelium structure and function. For example, one could co-pattern epithelial cells and goblet cells to understand how the distribution of different components of the epithelium influences overall tissue organization and function. Such information is critical for optimizing the design of a tissue-engineered epithelium for lining artificial organs. Furthermore our patterning system provides an interesting tool for drug screening. For example, in cell sheets with one cell type where a subset of cells are marked with GFP, observing pattern disruption provides a fast and easy readout to assess epithelial cell mobility within the cell sheet and the effect of particular treatments, such as drug treatments on this mobility.

Our patterning methodologies are adapted from two previously reported methods, originally developed for cell patterning on glass or culture plastic substrates [25, 27, 33]: a fibronectin microprinting method, which allowed us to produce co-patterned epithelial sheets with arbitrary features dependent on the design of the stamp, but requires the use of photolithographically produced stamps; and a parafilm insert method that allows the generation of stripe patterns only but is more easily adoptable by any lab since it does not require photolithography. We found that our fibronectin microprinting required significant optimization to ensure reproducible patterning and was sensitive to seeding cell density and timing of each step: Seeding at too low a cell density or incubation for too short a time results in incomplete coverage of the fibronectin stamped area (results not shown). Seeding at too high a cell density or incubating for too long a time resulted in cell clump formation and overgrowth of the cells beyond the patterned fibronectin stamped areas (results not shown). Both these scenarios lead to poor quality of cell patterning. For the particular cell types we used we found that different incubation times produced robust patterning, therefore, cell density and seeding times will likely have to be optimized for different epithelial cell types. Also, we found that the concentration of fibronectin solution was critical. Using less than $100 \mu \mathrm{g} / \mathrm{mL}$ fibronectin solution resulted in poor fibronectin printing. We speculate that this is due to poor confocal contact of the stamp with the porous membraneand therefore the fibronectin transfer or stamping is not as effective as on a rigid surface. Alternatively the fibronectin may not adsorb as effectively on the filter material compared to glass and tissue culture polystyrene. Using a higher concentration of fibronectin counteracts the effect of poor fibronectin transfer. The FBS incubation before the second cell type seeding was also very important to promote faster attachment of the second cell type. This avoided the migration of the first cell type out of the patterns.

We found the Parafilm insert method easier to implement but as with the fibronectin microprinting method it was important to optimize cell seeding densities and incubation times to ensure robust pattern generation. In the case of MDCK cells, if cells were seeded at too high a cell density or allowed to grow for too long then removal of the Parafilm insert without disrupting the epithelial sheets became very challenging. This was not a problem with ARPE-19 cells, which do not apically-basally polarize as quickly as the MDCK cells on the filters [37]. Depending on the epithelial cell type being patterned it is therefore important to establish when the insert can be removed without disruption of the cell sheet on the membrane. Other important parameters that influenced the quality of the co-pattern were (i) adequate bonding of the parafilm insert to the membrane's surface, (ii) the FBS wash step prior to seeding of the second cell type, and (iii) the cell density of the second cell type. We suggest using the degassing step in the insert preparation as a test for the strength of the bonding between parafilm and the membrane surface. If the parafilm detaches at this step even in some places, the probability of the successful patterning is very low. We also found the FBS wash step following the removal of the parafilm insert very important for promoting fidelity of the desired pattern. We speculate that FBS masks any cell-repellent parafilm residue left behind, promoting faster attachment of the second cell type to the areas free of the first cell type. In the absence of the FBS wash the first cell type started migrating out of the pattern before the second cell type had a chance to adhere, disturbing the imposed pattern (results not shown). Finally, with the parafilm insert method the success of the patterning depended more on the seeding density of the second cell type as opposed to that of the first cell type. Here, if the density of the second cell type was too low the first cell type migrated out of the pattern into the areas unoccupied by the second cell type, disrupting the pattern.

A key issue we needed to address for both methods was maintaining the co-culture patterns for an extended culture period (10-20 days) to allow adequate maturation of the epithelial sheets on the filter insert membranes. Pattern integrity over time varied depending on the motility of the epithelial cells and their tendency to move within the sheet. After 15 days in culture the co-culture patterns in MDCK 
Table 1. Comparison of Micropatterning Methods

\begin{tabular}{|l|l|}
\hline Fibronectin Microprinting Method & Parafilm Insert Method \\
\hline \hline Allows generation of arbitrary shape patterns & Pattern shapes are limited to stripes and circles [33]. \\
\hline Allows generation of arbitrary sizes of patterns & $\begin{array}{l}\text { Patterns are generated manually, and thus, are limited in size and precision by } \\
\text { experimentor`s ability to generate them }\end{array}$ \\
\hline $\begin{array}{l}\text { Requires access to photolithography equipment and knowledge of soft } \\
\text { lithography skills }\end{array}$ & Does not require any specialized equipment or set of skills \\
\hline Patterns can be maintained for 15 days, depending on the cell type & Patterns can be maintained for 15 days, depending on the cell type \\
\hline
\end{tabular}

cell sheets remained sharp suggesting little cellular rearrangement within the sheet occurs. Patterns in ARPE-19 cell sheets on the other hand became disrupted over the 15 day maturation period due to cell migration within the sheet. This increased migration behaviour may reflect the fact that ARPE-19 cells do not polarize as quickly as MDCK epithelial cells do over the 14-day culture period [37]. One potential strategy to decrease cell migration within the patterned sheets is to increase the adhesiveness of the filter substrate [38], for example by stamping with higher concentrations of fibronectin, to limit cell mobility on the filter surfaces. Assessing the ability of specific cell types to move within the sheet over time is therefore an important factor when designing an experiment that requires robust pattern maintenance.

Another key issue we wanted to assess was whether our co-patterning methods disrupted normal epithelial cells polarization and maturation. Both our patterning methods allow an even nutrient supply from the basal compartment of the filter insert to the epithelial layer; therefore we predicted that our techniques should have no effect on sheet polarization and maturation. To assess polarization we focused on MDCK cells, which polarize over 7-15 days on filters insert $[7,8]$ and form cilia on the apical surface of the cells. Cilia formation (number and timing of appearance) was not affected by our co-culture procedures, suggesting that epithelial sheet maturation was not affected by the coculture process.

Table 1 compares our two patterning methods and highlights the advantages and disadvantages of each. A consideration of these is important when selecting which method is most appropriate to address a specific biological question with the available resources. The fibronectin microprinting method is most useful when the epithelium sheet shape or pattern size are important factors for the experimental design. For example using this technique we could assess if the invasion of cancer cells into normal epithelial cell sheets depends on the size or the shape of the cancer cell sheet. We envision the parafilm patterning technique is most useful when the cell sheets size or pattern size are not important factors for the experimental design. Also, this method is appropriate when the use of protein such as fibronectin can affect the experimental outcome. For example using this technique we could assess the effect of a drug on pattern disruption rate and hence cell mobility within the epithelial sheet. Both co-culture systems are therefore powerful tools for understanding epithelial cell biology in vitro and for optimizing the design of tissueengineered epithelium.

\section{CONCLUSIONS}

Despite the importance of epithelial tissue in most major organs there have been limited attempts to tissue engineering artificial epithelium. A key feature of mature epithelium is the presence of an apical-basal polarization. We have developed a set of micropatterning techniques to spatially control the organization of co-cultures of epithelial cells on filter insert membranes under the culture conditions necessary to induce epithelial cell polarization. Each of our methods offers different advantages depending on the requirements of the system necessary for cell patterning. Our fibronectin microprinting method allows co-patterning of distinct populations of epithelial cells in any arbitrary pattern. Our Parafilm patterning method allows co-patterning distinct populations of epithelial cells in stripes but is extremely straightforward and could be adopted by any laboratory without the need of specialized microfabrication equipment and access to a clean room. Our methods will be useful for probing the importance of cell organization on epithelium function for applications in tissue engineering and generating relevant in vitro models of diseased epithelium.

\section{ACKNOWLEDGEMENTS}

This work was funded by a National Science and Engineering Research Council (NSERC) Discovery grant to AM and an NSERC CGS award to SJ. The authors have no conflict of interests to declare.

\section{CONFLICT OF INTEREST}

None declared

\section{REFERENCES}

[1] Kojima K, Vacanti CA. Generation of a tissue-engineered tracheal equivalent. Biotechnol Appl Biochem 2004; 39: 257-62.

[2] Tran SD, Wang J, Bandyopadhyay BC, et al. Primary culture of polarized human salivary epithelial cells for use in developing an artificial salivary gland. Tissue engineering 2005; 11: 172-81.

[3] Frank SA. Dynamics of Cancer: Incidence, Inheritance, and Evolution. 2010/09/08 ed. Princeton: Princeton University Press; 2007.

[4] Bissell MJ, Rizki A, Mian IS. Tissue architecture: the ultimate regulator of breast epithelial function. Curr Opin Cell Biol 2003; 15: 753-62.

[5] Bloom W, Fawcett DW. A Textbook of Histology 1968. 
[6] Freshney IR, Freshney MG. Culture of Epithelial Cells. Second ed. Glasgow: Wiley-Liss, Inc.; 2002.

[7] Wakabayashi Y, Chua J, Larkin JM, Lippincott-Schwartz J, Arias IM. Four-dimensional imaging of filter-grown polarized epithelial cells. Histochem Cell Biol 2007; 127: 463-72.

[8] Plachot C, Chaboub LS, Adissu HA, et al. Factors necessary to produce basoapical polarity in human glandular epithelium formed in conventional and high-throughput three-dimensional culture: example of the breast epithelium. BMC Biol 2009; 7: 77.

[9] Bhatia SN, Balis UJ, Yarmush ML, Toner M. Effect of cell-cell interactions in preservation of cellular phenotype: cocultivation of hepatocytes and nonparenchymal cells. FASEB J 1999; 13: 1883-900.

[10] Gibson MC, Perrimon N. Apicobasal polarization: epithelial form and function. Curr Opin Cell Biol 2003; 15: 747-52.

[11] Rosdy M, Clauss LC. Terminal epidermal differentiation of human keratinocytes grown in chemically defined medium on inert filter substrates at the air-liquid interface. J Invest Dermatol 1990; 95: 409-14.

[12] Handler JS, Preston AS, Steele RE. Factors affecting the differentiation of epithelial transport and responsiveness to hormones. Federation Proc 1984; 43: 2221-4.

[13] Plotnikova OV, Pugacheva EN, Golemis EA. Primary cilia and the cell cycle. Methods Cell Biol 2009; 94: 137-60.

[14] Bacallao R, Antony C, Dotti C, Karsenti E, Stelzer EH, Simons K. The subcellular organization of Madin-Darby canine kidney cells during the formation of a polarized epithelium. J Cell Biol 1989; 109: 2817-32.

[15] Jain R, Pan J, Driscoll JA, et al. Temporal relationship between primary and motile ciliogenesis in airway epithelial cells. Am J Respir Cell Mol Biol 2010; 43: 731-9.

[16] Praetorius HA, Spring KR. A physiological view of the primary cilium. Annu Rev Physiol 2005; 67: 515-29.

[17] Janvier R, Sourla A, Koutsilieris M, Doillon CJ. Stromal fibroblasts are required for PC-3 human prostate cancer cells to produce capillary-like formation of endothelial cells in a three-dimensional co-culture system. Anticancer Res 1997; 17: 1551-7.

[18] Lang SH, Smith J, Hyde C, Macintosh C, Stower M, Maitland NJ. Differentiation of prostate epithelial cell cultures by matrigel/ stromal cell glandular reconstruction. In Vitro Cell Dev Biol Anim 2006; 42: 273-80.

[19] Seltana A, Basora N, Beaulieu JF. Intestinal epithelial wound healing assay in an epithelial-mesenchymal co-culture system. Wound Repair Regen 2010; 18: 114-22.

[20] Zangani D, Darcy KM, Shoemaker S, Ip MM. Adipocyte-epithelial interactions regulate the in vitro development of normal mammary epithelial cells. Exp cell res 1999; 247: 399-409.

[21] Goke M, Kanai M, Podolsky DK. Intestinal fibroblasts regulate intestinal epithelial cell proliferation via hepatocyte growth factor. Am J Physiol 1998; 274: G809-18.
[22] Azadbakht M, Valojerdi MR, Mowla SJ. Development of mouse embryos co-cultured with polarized or non-polarized uterine epithelial cells using sequential culture media. Anim Reprod Sci 2007; 100: 141-57.

[23] Singer CF, Kronsteiner N, Marton E, et al. MMP-2 and MMP-9 expression in breast cancer-derived human fibroblasts is differentially regulated by stromal-epithelial interactions. Breast Cancer Res Treat 2002; 72: 69-77.

[24] Folch A, Ayon A, Hurtado O, Schmidt MA, Toner M. Molding of deep polydimethylsiloxane microstructures for microfluidics and biological applications. J Biomech Eng 1999; 121: 28-34.

[25] Bhatia SN, Yarmush ML, Toner M. Controlling cell interactions by micropatterning in co-cultures: hepatocytes and 3T3 fibroblasts. J Biomed Mater Res 1997; 34: 189-99.

[26] Jinno S, Moeller HC, Chen CL, et al. Microfabricated multilayer parylene-C stencils for the generation of patterned dynamic cocultures. J Biomed Mater Res A 2008; 86: 278-88.

[27] Peerani R, Bauwens C, Kumacheva E, Zandstra PW. Patterning mouse and human embryonic stem cells using micro-contact printing. Methods Mol Biol 2009; 482: 21-33.

[28] Nelson CM, Chen CS. Cell-cell signaling by direct contact increases cell proliferation via a PI3K-dependent signal. FEBS Lett 2002; 514: 238-42.

[29] Cho CH, Park J, Tilles AW, Berthiaume F, Toner M, Yarmush ML. Layered patterning of hepatocytes in co-culture systems using microfabricated stencils. BioTechniques 2010; 48: 47-52.

[30] Shen K, Qi J, Kam LC. Microcontact printing of proteins for cell biology. J Vis Exp : JoVE 2008.

[31] Whitesides GM, Ostuni E, Takayama S, Jiang X, Ingber DE. Soft lithography in biology and biochemistry. Annu Rev Biomed Eng 2001; 3: 335-73.

[32] Javaherian S, O'Donnell KA, McGuigan AP. A Fast and Accessible Methodology for Micro-Patterning Cells on Standard Culture Substrates Using Parafilm ${ }^{\mathrm{TM}}$ Inserts. PLoS One 2011; 6: e20909.

[33] Nauli SM, Alenghat FJ, Luo Y, et al. Polycystins 1 and 2 mediate mechanosensation in the primary cilium of kidney cells. Nat Genet 2003; 33: 129-37.

[34] Vieira OV, Gaus K, Verkade P, Fullekrug J, Vaz WL, Simons K. FAPP2, cilium formation, and compartmentalization of the apical membrane in polarized Madin-Darby canine kidney (MDCK) cells. Proc Natl Acad Sci USA 2006; 103: 18556-61.

[35] Praetorius HA, Spring KR. Removal of the MDCK cell primary cilium abolishes flow sensing. J Membr Biol 2003; 191: 69-76.

[36] Dunn KC, Aotaki-Keen AE, Putkey FR, Hjelmeland LM. ARPE19, a human retinal pigment epithelial cell line with differentiated properties. Exp Eye Res 1996; 62: 155-69.

[37] DiMilla PA, Stone JA, Quinn JA, Albelda SM, Lauffenburger DA. Maximal migration of human smooth muscle cells on fibronectin and type IV collagen occurs at an intermediate attachment strength. J Cell Biol 1993; 122: 729-37.

(C) Paz et al.; Licensee Bentham Open.

This is an open access article licensed under the terms of the Creative Commons Attribution Non-Commercial License (http://creativecommons.org/licenses/ by-nc/3.0/) which permits unrestricted, non-commercial use, distribution and reproduction in any medium, provided the work is properly cited. 